Meta

Journal des traducteurs

Translators' Journal

\title{
De la localisation à la délocalisation : enjeux professionnels
}

\section{Carmelo Cancio Pastor et Sydney Belmonte}

Volume 55, numéro 4, décembre 2010

De la localisation à la délocalisation - le facteur local en traduction

From Localization to Delocalization - The Local Factor in Translation

URI : https://id.erudit.org/iderudit/045684ar

DOI : https://doi.org/10.7202/045684ar

Aller au sommaire du numéro

Éditeur(s)

Les Presses de l'Université de Montréal

ISSN

0026-0452 (imprimé)

1492-1421 (numérique)

Découvrir la revue

Citer cet article

Cancio Pastor, C. \& Belmonte, S. (2010). De la localisation à la délocalisation : enjeux professionnels. Meta, 55(4), 661-673. https://doi.org/10.7202/045684ar

\section{Résumé de l'article}

Comme tout secteur de l'économie concurrentielle, celui de la localisation a subi les effets des délocalisations. Le présent article s'attache à observer les reflets que renvoie une activité professionnelle (la localisation) passée à travers le prisme d'un des faits économiques majeurs de notre temps (la délocalisation) : externalisation, expatriation, internationalisation, mondialisation... Il s'agit, dans un premier temps, de définir précisément ce qu'il faut entendre par localisation, avant d'examiner comment l'exercice concret de cette spécialité s'est trouvé et se trouve bouleversé par son quasi-antonyme. L'analyse critique de ces reflets permet d'identifier les enjeux et les tendances qui sous-tendent ce marché professionnel et leur influence sur celui de la traduction professionnelle dans son ensemble.
Ce document est protégé par la loi sur le droit d'auteur. L'utilisation des services d’Érudit (y compris la reproduction) est assujettie à sa politique d'utilisation que vous pouvez consulter en ligne.

https://apropos.erudit.org/fr/usagers/politique-dutilisation/ 


\title{
De la localisation à la délocalisation: enjeux professionnels
}

\author{
CARMELO CANCIO PASTOR \\ Université Toulouse II-Le Mirail, Toulouse, France \\ carmelo@cancio.com \\ SYDNEY BELMONTE \\ Université Paris Diderot - Paris 7, Paris, France \\ sydney.belmonte@orange.fr
}

\begin{abstract}
RÉSUMÉ
Comme tout secteur de l'économie concurrentielle, celui de la localisation a subi les effets des délocalisations. Le présent article s'attache à observer les reflets que renvoie une activité professionnelle (la localisation) passée à travers le prisme d'un des faits économiques majeurs de notre temps (la délocalisation): externalisation, expatriation, internationalisation, mondialisation... II s'agit, dans un premier temps, de définir précisément ce qu'il faut entendre par localisation, avant d'examiner comment l'exercice concret de cette spécialité s'est trouvé et se trouve bouleversé par son quasi-antonyme. L'analyse critique de ces reflets permet d'identifier les enjeux et les tendances qui soustendent ce marché professionnel et leur influence sur celui de la traduction professionnelle dans son ensemble.
\end{abstract}

\begin{abstract}
Like any market sector, the localization industry has had to accommodate the effects of delocalization. This article is an attempt to observe the reflections sent out by a specific industry (localization) which has passed through the prism of a major economic trend (delocalization): externalization, expatriation, internationalization, globalization... We first try to define precisely what is to be understood by the word localization, and then look at the manifold ways this profession has been changed though the incidence of its quasiantonym. The critical analysis of these reflections helps to identify the challenges and trends which underpin this professional market and their influence on the professional translation market as a whole.
\end{abstract}

\section{MOTS-CLÉS/KEYWORDS}

localisation, délocalisation, mondialisation, marché de la traduction localization, delocalization, globalization, translation market

La thématique du présent numéro, De la localisation à la délocalisation - le facteur local en traduction, est une invitation au voyage. Voyage à la découverte de la localisation, d'abord, car le périmètre de cette activité professionnelle a considérablement varié avec le temps. Voyage sur les terres de la mondialisation, ensuite, car ce phénomène économique a bouleversé - et continue de bouleverser - l'organisation de cette profession et, au-delà, celle de traducteur en général. Précisons d'emblée que cet article est l'œuvre d'un professionnel de la localisation devenu enseignant-chercheur et soucieux d'ancrer sa réflexion théorique dans l'expérience: quelles sont les spécificités du marché de la localisation, ses contraintes, son évolution, ses pratiques et, comment celles-ci, débordant le milieu informatique, façonnent-elles désormais les 
marchés de la traduction dans leur ensemble? Cette approche nous amènera parfois à faire état de phénomènes observés sur le marché ou dans les discussions entre praticiens et pour lesquels il n'y a donc pas obligatoirement de sources bibliographiques. Nous prions par avance le lecteur de nous en excuser, mais nous pensons que c'est le prix intellectuel à payer pour une prise en compte aussi précise que possible par la recherche des phénomènes observés sur le terrain. Nous pensons ici, et dans notre domaine spécifique, être fidèles à l'esprit d'Antoine Berman, qui définissait la traductologie comme «la réflexion de la traduction sur elle-même à partir de sa nature d'expérience» (Berman 1985/1999: 16-17).

\section{Comment situer la localisation?}

Localisation est un terme qui pose problème, car, comme nous le verrons, il est souvent utilisé dans un sens qui nous paraît beaucoup trop large. Il n'est pas aisé de cerner le domaine qu'il couvre, ni de déterminer précisément de quoi il s'agit. Commençons par observer que le français localisation est, pour tous les professionnels, considéré comme équivalant à l'anglais localization, même si d'autres termes (adaptation ${ }^{1}$, qu'il est aisé de confondre avec la traduction audiovisuelle, ou adaptation locale ${ }^{2}$, notamment) ont pu, par le passé, être proposés. Il y a en tout état de cause une difficulté à cerner ce qu'il faut entendre par localisation. Difficulté qui ne se pose pas véritablement pour les professionnels (qui, comme nous le verrons dans la deuxième partie de cet article, sont confrontés à d'autres soucis). En effet, comme l'observe Anthony Pym, "The discourse of localization has not developed from translation theory, nor is it an invention of academic linguistics, as far as I know. It comes from practice [...]» (Pym 2003: 296). Paradoxalement, ce n'est pas en partant de la définition donnée par la LISA (The Localization Industry Standards Association) que nous parviendrons à surmonter cet obstacle: "Localization is the process of modifying products or services to account for differences in distinct markets ${ }^{3}$.»

C'est bien vague: de quels produits, de quels services s'agit-il ? L'absence de précision nous amène à penser que tous sont concernés. Mais c'est précisément ce caractère englobant qui peut nous mettre sur la piste des véritables enjeux épistémologiques et économiques d'une telle démarche. En effet, cette définition émane d'une association professionnelle des entreprises de localisation. Or, la croissance des entreprises passe souvent par le développement de leurs activités hors de leur sphère d'origine. Et effectivement, la localisation a d'abord (fin des années 1980) concerné les logiciels, avant de s'élargir à d'autres activités et services. Il est symptomatique à cet égard qu'un des ouvrages de référence sur le sujet, signé par Bert Esselink, ait perdu le substantif software dans son titre entre sa première et sa deuxième édition: en deux ans seulement, nous sommes passés de A Practical Guide to Software Localization (1998) à A Practical Guide to Localization tout court (2000). Pour d'autres ouvrages de référence, le lecteur pourra aussi se référer à Smith-Ferrier (2006), ou DePalma (2004). On retrouve ce décalage aux premières pages de l'édition $2000 \mathrm{du}$ même ouvrage. Celles-ci passent en effet de:

Generally speaking, localization is the translation and adaptation of a software or web product, which includes the software application itself and all related product documentation. (Esselink 2000: 1) 
À la définition qui était alors donnée par la LISA et qui, déjà, avait cessé de mentionner spécifiquement les logiciels:

Localization involves taking a product and making it linguistically and culturally appropriate to the target locale (country region and language) where it will be used and sold. (Esselink 2000: 3)

À la différence d'une étude antérieure, qui ancrait bel et bien la localisation dans le secteur du programme informatique:

Localization is the process of adapting a product, in our context a software program, to a specific locale, i.e., to its language, standards and cultural norms as well as to the needs and expectations of a specific target market. A properly localized product also meets all the legal requirements in force in the user's region. (Dohler 1997)

Cette définition ancienne rejoint au demeurant celle donnée aujourd'hui par la société Microsoft, qui présente la localisation comme une des facettes de la création de logiciels internationalisés ${ }^{4}$ (nous reparlerons de cet aspect au point 2.1).

De prime abord, il n'y a là rien que de très banal: les notions évoluent avec le temps. Nous pensons néanmoins que cette explication par le glissement de sens est loin d'épuiser le sujet. En effet, à travers le dilemme entre, d'un côté, logiciel et, de l'autre, produits et services, il s'agit à notre sens de déterminer s'il faut voir dans la localisation une composante de la traduction (ce qui est l'approche généralement retenue par les formations universitaires), ou bien l'inverse (comme peuvent le penser ceux qui estiment que le terme de traducteur est dépassé et devrait être remplacé par un autre: ingénieur linguiste, spécialiste en communication multilingue, langagier, localisateur, localiseur...), ou encore si l'on a affaire à deux aspects distincts d'une même réalité, elle-même exposée aux vents de la mondialisation. Sans oublier que cette relation se noue sur un marché concurrentiel (très concurrentiel, même) et relève donc du rapport de force économique - et, au-delà, du politique: comme l'écrit Yves Gambier (2006: 849) au sujet, justement, de la mondialisation, il importe de nommer les choses. En ce qui concerne la localisation, il est symptomatique que beaucoup des auteurs qui cherchent à cerner ce concept le fassent d'une manière que nous qualifierions de louvoyante, au point qu'il soit possible de leur faire dire une chose et son contraire. Il nous faut pourtant essayer. Au risque de simplifier, donc, une situation complexe et mouvante, trois cas de figure nous semblent se dessiner.

\subsection{La localisation comme sous-ensemble de l'activité de traduction}

L'hésitation que nous venons de mentionner est bien rendue par Jean Quirion (2006), qui commence par observer que la localisation, dont le chiffre d'affaires, au moment où il rédige son article, commence à rivaliser avec celui de la traduction, ne représente qu'une «accentuation» (Quirion: 826) d'un mouvement d'adaptation textuelle séculaire, avant de préciser:

La définition de la localisation tend d'ailleurs à s'étendre, à se modifier progressivement pour inclure la somme des ordres de produits, informatiques ou non, qui sont matière à une conversion linguistique et culturelle. Cette conversion, qu'on l'appelle adaptation ou localisation, est rentable et le demeurera tant pour les biens informatiques que pour les autres genres d'articles. (Quirion: 826; les italiques sont de l'auteur) 
En somme, née du secteur informatique, la localisation tendrait à rejoindre la traduction. Pour le plus grand bonheur économique des traducteurs:

[d]'aucuns langagiers ont à leur tour vu dans la localisation une chance inespérée de faire un bon coup de commercialisation, c'est-à-dire une occasion de mieux vendre (et plus cher!) de la bonne vieille traduction. (Quirion 826)

Certes, dans la phrase qui suit, l'auteur nuance son propos:

C'est en partie vrai peut-être, mais la localisation annonce bien davantage qu'un avatar de forme: un de ses mérites, et non des moindres, est une remise en cause des paradigmes de la traduction, comme celui de la préséance du texte de départ. (Quirion 826)

Mais cet argument est-il suffisant pour attester de l'autonomie de la localisation par rapport à la traduction? Ce n'est pas notre impression, car la remise en cause de la primauté du texte de départ se trouve aussi et déjà dans la théorie fonctionnaliste, pour laquelle l'activité de traduction est tout entière tendue vers la production d'un texte d'arrivée qui fonctionne dans son contexte précis de réception (Vermeer 1996). Sans parler des Belles infidèles de l'âge classique... Dans cette première vision de la relation traduction/localisation, cette dernière apporte donc bel et bien une nouveauté, mais celle-ci est finalement réintégrée dans le vaste ensemble de la traduction. Transposé au domaine pédagogique, ce point de vue revient à dire qu'enseigner la traduction, c'est déjà (nonobstant les aspects informatiques sur lesquels nous reviendrons plus loin) enseigner la localisation.

\subsection{La localisation comme aboutissement de l'activité de traduction}

On trouve un point de vue symétrique chez Daniel Gouadec (2003). Pour cet auteur, il semble exister une relation dialectique dans laquelle la localisation apparaît comme le lieu où trouvent à s'accomplir les compétences les plus pointues du traducteur:

La connaissance des contenus de la prestation de localisation permet de construire le profil du localiseur ou localisateur vu ici comme le traducteur le plus abouti en termes de maîtrise de la traduction spécialisée, de la rédaction et de la réécriture, de contrôle des ergonomies, de gestion de la qualité, de gestion de projets, de gestion d'équipes de projets et, bien évidemment, de maîtrise de l'informatique vue à la fois comme une discipline, comme une technique, et comme une somme d'outils. (Gouadec 2003: 526; souligné par l'auteur)

L'auteur propose alors deux options. Dans la première, le traducteur n'est qu'un intervenant dans une chaîne qui comprend en outre concepteurs, développeurs, graphiques, rédacteurs, ainsi que divers autres spécialistes et techniciens (Gouadec 2003: 528-529). Dans la seconde, il se doit, pour capter une part aussi conséquente que possible de la valeur ajoutée, d'élargir ses compétences à tout cet ensemble:

[1]e localiseur/localisateur est le traducteur le plus abouti avec des compétences particulières (additionnelles) le rendant apte à la pratique spécifique de la localisation. Il connaît les matériaux particuliers qui font l'objet de la localisation, il maîtrise les environnements requis, il connaît et sait gérer des partenaires nouveaux et plus présents que dans les situations habituelles de traduction. Mais il s'agit bien d'un traducteur. (Gouadec 2003: 536) 
Au paragraphe 1.1, le localisateur se faisait traducteur; ici, c'est le traducteur qui est invité à devenir localisateur. Et cela pour éviter de se trouver marginalisé - y compris économiquement - dans la chaîne de production. Et là encore, en termes d'enseignement, on en déduira qu'une pédagogie de la localisation est la potion qui convient aux futurs langagiers si l'on tient à ce que ceux-ci parviennent à vivre correctement de leur profession. Les compétences de ce que l'auteur appelle le «traducteur standard» (Gouadec 2003: 535), celui de la première option, sont naturellement un des ingrédients du breuvage. Ce point de vue est également (nonobstant une nuance sur la spécificité et la nouveauté de la localisation en tant que lieu d'une «interculture» [Pym 2003: 300]) celui d'Anthony Pym.

Querelles de territoires, donc: on voit l'articulation traduction/localisation dans des sens opposés selon qu'on l'observe du point de vue de la première ou de la seconde. Et les tentations annexionnistes qui en découlent sont bien naturelles. Quel est alors le point de vue des professionnels?

\subsection{Localisation et traduction sont deux opérations bien distinctes}

Nous nous rangeons pour notre part, et sur la base de notre expérience, parmi ceux qui voient une réelle différence entre localisation et traduction (avec les différents degrés d'adaptation applicables à chacune d'entre elles). Et cette différence nous paraît liée à la nature de l'activité, aux compétences mobilisées pour l'accomplir et à sa finalité. Pour le comprendre, nous devons nous intéresser à la genèse du terme.

À l'origine du terme et de ses dérivés (notamment le verbe localiser) se trouve le substantif anglais locale (Esselink 2000: 1), qui renvoyait initialement à l'idée d'un lieu spécifique: "a place where something happens or is set, or that has particular events associated with it ${ }^{5}$ ». Il s'agit, au sens technique qu'a pris ce terme avec le développement de l'intelligence artificielle, des paramètres de configuration utilisés par les informaticiens pour qu'un produit logiciel fonctionne convenablement dans un endroit donné. Ces paramètres de lieu sont le plus souvent réunis dans un fichier de configuration. Ils précisent aussi bien la langue (et, éventuellement, la variante linguistique) qui doit être employée pour afficher les éléments textuels de l'interface du logiciel (menus, boîtes de dialogue, messages d'erreur...) que les réglages nécessaires à la bonne interprétation des chiffres (conventions d'écriture des nombres, des dates ou des heures, monnaie utilisée), le tri correct des textes et des nombres... mais aussi le type de clavier utilisé ou les protocoles de télécommunication en vigueur dans le pays de l'utilisateur. Par conséquent, l'ensemble des paramètres de lieu représente pour le développeur une sorte de cahier des charges à respecter pour que l'utilisateur final puisse se servir convenablement du logiciel considéré ${ }^{6}$.

À partir de cet ancrage concret, la localisation peut être définie comme la mise en conformité d'un logiciel avec ces paramètres pour un lieu donné ou, en d'autres termes, l'adaptation de l'interface et du fonctionnement d'un logiciel au marché de destination. Sa spécificité est que, à la différence de la traduction, elle ne porte pas sur un document, mais sur un programme, dont la finalité n'est pas de communiquer mais de faire. C'est une forme aiguë de la performativité.

Certes, un programme, qui n'est rien d'autre qu'une série de commandes déclenchant des actions exécutées par un ordinateur, s'appuie sur une interface pour permettre à l'utilisateur de s'en servir. Certes, cette interface contient du texte présenté 
suivant certaines conventions qui, dans le cas des interfaces graphiques modernes, sont dictées par l'exiguïté de l'espace d'affichage et des fonctionnalités spécifiques liées à leur emplacement (le titre d'une boîte de dialogue, le texte d'un bouton ou celui d'un menu obéissent à des règles rédactionnelles différentes). Mais ce qu'il faut retenir, c'est que le contenu textuel d'une interface n'est qu'une composante de celleci, laquelle n'est à son tour qu'une composante du logiciel, dont la partie fondamentale est formée par des milliers de lignes de code invisibles pour l'utilisateur. La localisation d'un logiciel exige donc, par voie de conséquence mais néanmoins de façon subsidiaire, l'adaptation de l'ensemble de ses composantes aux exigences conventionnelles posées par l'environnement de l'utilisateur final.

Dans ce contexte, la traduction ne saurait, de notre point de vue, être confondue avec la localisation dont elle n'est, dans le domaine considéré, qu'une facette. Cette observation ne doit pas revenir à minimiser le rôle du traducteur, mais bien au contraire à mettre en valeur sa place au sein d'une équipe pluridisciplinaire. En effet, le processus de localisation comprend:

- La modification de l'interface du logiciel: traduction ou adaptation des éléments textuels, redéfinition des raccourcis clavier, ajustement de la taille et de la disposition des composants graphiques de l'interface (fenêtres, menus, boîtes de dialogue, boutons...), etc.;

- L'adaptation fonctionnelle du logiciel pour qu'il puisse traiter les données conformément aux conventions et aux normes en vigueur dans le marché d'arrivée: tris textuels et numériques; format des nombres, des dates et des heures; saisie, capture, importation, exportation et transfert de données...;

- Le test du produit dans l'environnement de l'utilisateur (version linguistique du système d'exploitation; configuration matérielle; périphériques d'entrée, de sortie et de communication...).

La part dévolue à la traduction au sens le plus traditionnel du terme constitue ainsi - et, nous le répétons, dans le cadre précis de la spécialité professionnelle de localisateur - une étape ou une partie, évidemment essentielle, du processus de localisation qui mobilise d'autres compétences, telles que la conduite de projets multilingues, l'ingénierie de localisation ou l'ingénierie de développement. Si, comme on vient de le voir, la localisation fait intervenir des aptitudes qui vont au-delà de la traduction pure, la confondre avec des notions réductrices (la traduction des interfaces des logiciels) ou, au contraire, exagérément élargies (la traduction dans le domaine informatique) est la manifestation d'un premier égarement notionnel, d'un premier décalage auquel il nous est apparu important de remédier. C’est désormais chose faite. Mais, ce premier décalage n'est-il pas annonciateur d'autres déplacements, d'autres recompositions territoriales? Cette question va nous amener à nous pencher sur les évolutions du métier de localisateur à mesure des progrès de la mondialisation et des délocalisations.

\section{La délocalisation et ses conséquences sur le secteur}

Au sens propre, le terme délocalisation exprime la notion de changement d'emplacement, de déplacement, de décalage. C'est la perte du caractère local pour un produit (émergence de marques mondiales), une activité (télétravail) voire, mais ce sens est plus rare, une acception, un sens. Et bien sûr, ce terme a pris au cours des dernières 
décennies, un sens micro- et macroéconomique: celui du départ des activités de production d'entreprises des pays développés vers le tiers-monde, considéré à la fois comme gisement de main-d'œuvre bon marché et comme relais de croissance, ces deux aspects s'engrenant l'un sur l'autre.

Le secteur du logiciel n'a pas été épargné par cette tendance, au point que certains peuvent être tentés de risquer un jeu de mots entre localisation et délocalisation:

This would seem to be the main message of software localization: investments in a generic product mean savings in multiple locales. Admittedly, 'internationalization' is not a happy term for this generic product - nations have little to do with this - and one might talk more fashionably about 'delocalization' as the step to the middle stone. (Pym 2003: 298)

Un tel renversement était-il d'emblée inscrit, semblait-il relever d'un processus naturel dans le domaine de la localisation de logiciels? Apparemment non, car le cahier des charges fonctionnel constitué par les paramètres de lieu est censé agir comme garde-fou contre une telle inclination: la localisation consistait au départ à adapter des logiciels à des marchés locaux. De plus, la logique commerciale impose a priori de vendre un produit qui «marche» dans le double sens du terme, c'est-à-dire qui remplisse sans encombre les tâches pour lesquelles il a été créé et qui se vende bien. On sait, dans le même temps, que la mondialisation constitue un processus d'effacement graduel des frontières nationales. Il nous paraît ici intéressant d'envisager comment ce phénomène a renversé une à une les barrières à l'intérieur desquelles s'était initialement édifié le marché de la localisation.

\subsection{Dématérialisation: le producteur prend le pas sur le consommateur}

Les éditeurs de logiciels ont tout d'abord rapidement compris qu'il fallait trouver des parades aux obstacles technologiques ou méthodologiques qui rendaient difficile, sinon impossible, la déclinaison d'un même logiciel en des variantes linguistiquement et culturellement adaptées à différents marchés. Est alors née, dans le contexte anglophone, l'internationalisation (Esselink 2003; Niosi et Tschang 2009) qui, anticipant les écueils, permettait de prévoir dès la conception d'un logiciel des solutions aux contraintes spécifiques qu'introduirait sa localisation. On peut par exemple utiliser pour cela des architectures logicielles telles que le Model-View-Controller (MVC), qui permet d'isoler données d'entrée, logique applicative et présentation, et ainsi d'intervenir séparément sur ces trois couches d'un même produit logiciel. Fini le temps où il fallait développer et maintenir deux versions d'un même logiciel en tous points identiques sauf dans le recours à la technologie permettant de coder les caractères: une version qui reproduisait les caractères des langues occidentales et une autre pour les idéogrammes des langues orientales. Fini le temps où il fallait se plonger dans des milliers de lignes de code de programmation à la recherche des chaînes de caractères ou messages qu'il fallait traduire, au risque, par la suppression malencontreuse de la moindre virgule, d'introduire des bogues ou dysfonctionnements dans le logiciel. Un logiciel internationalisé devenait ainsi un logiciel prêt à être localisé.

Mais à cette logique commerciale, tournée vers l'utilisateur final, est vite venue s'opposer l'implacable logique économique du producteur. Dans sa recherche incessante d'une plus grande rentabilité, celui-ci a trouvé des moyens de réduire les coûts de production, avec pour effet d'affecter le travail du traducteur: la dématérialisation, 
c'est-à-dire la disparition, dans un premier temps, des supports papier puis des CDROM accompagnant les logiciels, a permis d'économiser les frais d'impression des documentations qui, passant de l'écrit à l'écran, sont devenues des documents électroniques, ainsi que les frais de pressage des CD-ROM des logiciels, que l'on se procure maintenant de plus en plus fréquemment par téléchargement. Ce phénomène ne se limite d'ailleurs pas à la localisation, mais s'étend au contraire à bien d'autres activités de traduction. Le traducteur n'a donc plus (ou a de moins en moins) de documentation physique à traduire et les spécificités de lecture des documents électroniques modifient substantiellement la nature des documentations techniques accompagnant les logiciels (voir par exemple Mehedintu et Pirvu 2009) et sur lesquelles il est censé intervenir.

\subsection{Du plurilinguisme à l'anglais international}

Forts du constat que de plus en plus de personnes utilisaient l'anglais comme langue d'échange internationale, indépendamment de leur pays de résidence, certains éditeurs n'ont alors pas hésité à concevoir une seule édition internationale de leurs logiciels (en anglais, bien sûr) destinée à être vendue dans le monde entier, réduisant les coûts de localisation au minimum indispensable (le paramétrage fonctionnel). Seule une adaptation minimale devenait nécessaire!

D'autres, plus audacieux encore, constatant que leurs programmeurs étaient habitués à se documenter en anglais et à utiliser des langages de programmation où cette langue est omniprésente, n'ont pas été longs à conclure que la maîtrise de l'anglais était acquise dans ce contexte et que les interfaces des logiciels pouvaient être rédigées directement dans cette langue. L’adaptation n'était même plus nécessaire!

Il n'est pas rare, aujourd'hui, de trouver dans tous les pays du monde des éditeurs ayant des ambitions internationales qui élaborent directement - voire uniquement - en anglais leurs logiciels. Ce n'est bien sûr pas le cas de la totalité des éditeurs de logiciels. Ainsi, les versions multilingues restent la règle pour les applications grand public et les systèmes d'exploitation. En revanche, là où l'usage exclusif de l'anglais se rencontre, la limitation terminologique, la pauvreté expressive et le manque de clarté qui caractérisent le produit d'arrivée ne passent pas inaperçus de l'utilisateur final (qu'il soit de langue maternelle anglaise ou pas) - ni, malheureusement, du traducteur lorsque celui-ci se trouve amené à intervenir à la périphérie de ce produit internationalisé. Encouragés, certainement, par l'exemple de l'anglais dit international, de plus en plus présent dans le milieu de la communication professionnelle (une seule version anglaise des supports de communication, où l'on tend à gommer tout régionalisme et à simplifier au maximum les structures grammaticales de façon à ce que les documents puissent être lus par tout anglophone, natif ou pas), ces éditeurs se contentent de logiciels aux interfaces maladroitement rédigées dans un anglais appauvri. Nous ne sommes pas très loin de ce que Jean-Paul Nerrière (2004-2006 et 2005) a baptisé globish. Visionnaire ou précurseur, cet ancien vice-président d'IBM pour les États-Unis a su nommer et caractériser en 1989 une tendance qui allait se confirmer par la suite: celle de la dilution des frontières nationales des communautés linguistiques des langues à grande diffusion. Une certaine idée de la mondialisation, négatrice des identités nationales et de leurs manifestations expressives à travers les variantes linguistiques d'une même langue trouvait alors ses lettres de noblesse dans 
les entreprises multinationales. C'est cette idée particulière de la mondialisation qui apparaît comme un nouveau décalage, une nouvelle délocalisation qui porte atteinte aux intérêts des utilisateurs finals et, pour ce qui nous concerne, au marché de la localisation.

Si cette tendance s'est manifestée initialement dans le domaine de l'édition de logiciels et, par extension, des jeux vidéo, elle s'est ensuite imposée sur Internet où les deux notions, localisation et délocalisation, campent de toute évidence sur des positions opposées: les sites multilingues des institutions et des entreprises tranchent nettement la question en optant soit pour des sites localisés en une ribambelle de versions linguistico-culturelles, soit pour des sites proposant uniquement deux langues, celle du pays d'origine de l'entreprise et l'anglais international évoqué plus haut.

\subsection{De l'externalisation à l'expatriation}

Dans le même temps, la recherche de la rentabilité, le fait que les compétences requises par la localisation soient essentiellement intellectuelles et le développement des technologies de l'information et de la communication (TIC), avec en particulier l'Internet à haut débit, ont contribué à déplacer, cette fois-ci physiquement, les services de localisation des entreprises éditrices de logiciels. On a assisté là à une délocalisation au sens propre qui a connu deux phases: l'externalisation, tout d'abord, l'expatriation ensuite.

Les équipes de développement de logiciels, de jeux vidéo et d'applications exploitant les technologies Internet ont été amputées de leurs services de localisation, désormais sous-traités à des prestataires externes. Perçus comme ne faisant pas partie du cœur de métier de l'édition de programmes informatiques (comment expliquer, sinon, qu'ils aient pu être ainsi écartés des centres névralgiques de production?), les services de localisation se sont alors développés au sein de sociétés de traduction qui en ont fait leur spécialité. Ce phénomène s'apparente donc fortement à une tendance qu'a connue le marché de la traduction à plusieurs reprises depuis la fin des années 1980 (avec aussi des revirements occasionnels), à savoir l'externalisation de tout ou partie de la fonction traduction dans les grandes entreprises. Une recherche rapide sur Internet montre ainsi que les termes externalisation et traduction renvoient d'abord à quelques ressources terminologiques (comment traduire externalisation en anglais) et ensuite avant tout à des grandes agences de traduction et de localisation.

Cette évolution a donc favorisé l'éclosion d'une activité qui a connu un développement spectaculaire au cours des 30 dernières années, jusqu'à devenir le segment le plus porteur parmi les métiers des langues: 26 des 30 plus grandes entreprises de traduction du monde opèrent aujourd'hui dans la localisation (Beninatto et Kelly 2009: 3, tableau 3).

Or, nous l'avons vu, toute entreprise de localisation fait aussi de la traduction - ne serait-ce que des manuels des logiciels qu'elle localise - et les plus grandes d'entre elles ont profité de l'élan que la localisation a donné à leurs activités pour se diversifier et s'implanter dans le monde entier. Leurs méthodes de travail, leur philosophie, leurs pratiques, profondément modelées par les contraintes du monde de la localisation, moteur de leur prospérité, ont imprégné toutes les facettes de leurs activités et fini par s'imposer: utilisation à outrance d'outils d'aide à la localisation ou à la traduction et 
de traduction automatique, dépersonnalisation du travail par la mise en commun des traductions réalisées dans des bases de données collectives (mémoires de traduction), calibrage et organisation du travail de traduction déterminés par les analyses statistiques proposées par les outils de TAO (qui se mesure en termes de «mots à traduire» et de "mots à réviser», ces derniers s'échelonnant en fonction de leur pourcentage de fiabilité pour composer un large barème de temps/tarifs), éclatement des processus en tâches déliées de leur contexte, traduction ou révision à l'aveugle de "chaînes de texte» ou de "segments de texte», etc. (voir Gouadec 2002, Froeliger 2007): tel est aujourd'hui le quotidien d'une large proportion des localisateurs et des traducteurs.

Si l'externalisation a eu lieu dans un premier temps chez les éditeurs de logiciels, elle s'est en outre reproduite une deuxième fois chez les prestataires de services, à quelques années d'intervalle. Profitant des possibilités de télétravail qu'offraient les nouvelles technologies, ces entreprises de traduction et de localisation ont à leur tour sous-traité leur production, gardant dans leurs agences de plus en plus de gestionnaires et de moins en moins de traducteurs. Ce modèle de fonctionnement a pour conséquence l'éclatement des équipes et des processus de localisation, les compétences de développement restant chez le client, les compétences de gestion chez l'entreprise de localisation et celles de traduction chez le prestataire final (voir St. Amant 2005 et Coris 2008). La taylorisation de la profession - qui n'est certainement pas un gage d'enrichissement intellectuel et monétaire pour les exécutants - est bien avancée.

Les effets de cette délocalisation des compétences de localisation ont été dévastateurs pour les traducteurs intervenant en bout de chaîne de sous-traitance, dont les conditions de travail et de rémunération ne cessent de se dégrader, mais aussi pour la qualité des résultats obtenus en raison d'une perte de cohésion et de cohérence dans les méthodes, les processus et les ressources de localisation mobilisées. C’est, élargi à la structure de la profession ${ }^{7}$, le phénomène décrit et condamné, à l'échelle des textes, par Henri Meschonnic (2006: 127-128, par exemple) lorsqu'il traite du passage du continu au discontinu, ou, ailleurs (1999), du signe au rythme. C'est aussi une des raisons pour lesquelles les associations de traducteurs et de localisateurs ont tendance à se développer depuis quelques années : il y a lieu de défendre ces professions... Comme les praticiens l'ont constaté ailleurs en traduction, les calculs de gestionnaire ne font pas toujours bon ménage avec la qualité ou avec un environnement de travail gratifiant. Dans ces conditions, il est légitime de se demander si la délocalisation des activités de localisation est réellement efficace et rentable à long terme. Sur ce point, nous ne sommes pas aussi optimistes que Debbie Folaron et Yves Gambier, qui voient dans les retombées actuelles de la mondialisation économique une réappropriation par la base (les utilisateurs finals) des compétences et des mécanismes de la localisation (Folaron et Gambier 2007: 42; voir aussi notre point 2.1).

D’abord national, ce double phénomène d'externalisation a vite dépassé les frontières étatiques. La concurrence, ainsi que la mondialisation des échanges et des marchés favorisée par les technologies de la communication, caractérisent cette nouvelle vague de délocalisations: est alors arrivée l'expatriation des services soustraités. Aujourd'hui, un logiciel peut très bien être conçu en France, développé en Inde et localisé dans 20 pays différents sous la responsabilité d'un chef de projet qui, lui, peut être basé en Irlande. Une fois de plus, nous retrouvons le concept de mon- 
dialisation et, avec lui, un nouveau décalage paradoxal: le marché de la localisation est bel et bien devenu une des composantes de la mondialisation.

\subsection{Extension du domaine de la localisation}

Pour autant, ce décalage n'est pas le dernier qui caractérise ce marché. En effet, au déplacement physique des services de localisation et à celui du cœur de métier - de la localisation vers la mondialisation - s'ajoute un glissement (un élargissement, à proprement parler) vers d'autres secteurs d'activité. La localisation n'est plus, désormais, cantonnée au seul domaine des applications informatiques. Après s'être étendue aux domaines connexes d'Internet, du multimédia et des jeux vidéo (qui, rappelonsle, ont une composante purement logicielle), elle s'applique maintenant à tous les domaines qui font appel à l'électronique pilotée: depuis le cinéma (en salle ou à domicile, pourvu qu'il soit numérique) jusqu'à l'édition électronique, en passant par l'électroménager grand public ou l'automobile, on trouve partout des interfaces logicielles - qu'il a fallu localiser: c'est la réalité des praticiens d'aujourd'hui. Et cela explique en partie les hésitations évoquées dans notre première partie quant à la délimitation précise de la localisation.

Un dernier déphasage, et non des moindres, illustre à quel point ce secteur des métiers de la traduction est en décalage par rapport au reste: en pleine période de récession économique mondiale, le marché de la localisation est en plein essor. Lors de la TCWorld Conference organisée par l'association professionnelle GALA (Globalization and Localization Association) en 2008, le directeur exécutif de cet organisme faisait état de la prospérité de ce marché, à contre-courant de la conjoncture économique, en s'appuyant sur les résultats d'une étude récente, résumée par Hollan $\left(2008^{8}\right)$ :

- Plus de $85 \%$ des membres de GALA avaient vu leur chiffre d'affaires augmenter en 2007 (dont la moitié, de plus de $20 \%$ );

- Plus de $75 \%$ des membres de GALA avaient augmenté leurs effectifs en 2007 (dont $25 \%$ de plus de $20 \%$ );

- Les investissements en outils technologiques avaient augmenté de $39 \%$ parmi les membres de GALA en 2007.

De leur côté, les analystes de Common Sense Advisory estimaient en 2009 le rythme de croissance du marché à 10,76\% par an pour les cinq années suivantes, et constataient - c'est une des raisons d'espérer, après les tendances lourdes décrites au point 2.1 - une augmentation sensible du nombre de langues dans lesquelles les produits étaient localisés (Beninatto et Kelly 2009: 1).

\section{Conclusion}

La délocalisation sous ses différentes déclinaisons compose donc un paysage attractif pour les entrepreneurs mais désolant pour les traducteurs œuvrant dans la localisation. Qui plus est, la puissance des grandes entreprises de localisation est en train de façonner à leur avantage l'offre du marché, représentée par les producteurs que sont les traducteurs.

Les enjeux sont de taille. Tout d'abord pour les traducteurs concernés, relégués à un rôle de second plan. Mais aussi pour la qualité des produits localisés, menacés 
par l'éclatement des processus, des équipes et des méthodes. On ne peut, ici, qu'espérer que les phénomènes récents de relocalisation et de ré-internatisation des activités de production, motivés par des préoccupations de qualité, de fiabilité et de stabilité des produits, se communiquent également à la localisation. Et c'est une des tâches des traducteurs et localisateurs d'aujourd'hui que de se faire les avocats de telles stratégies. C'est une vision volontariste.

À défaut, on peut se demander, à la lumière de l'évolution du marché, si la technophilie dominante parmi les donneurs d'ordre n'est pas un danger pour les compétences traductionnelles, si nous ne sommes pas en train d'assister à une modification profonde de la structuration du marché de la traduction dans son ensemble, si les impératifs de la mondialisation et les facilités des nouvelles technologies, enfin, ne poussent pas in fine une partie de la production localisée à la déprofessionnalisation.

En effet, le corollaire de cette tendance où localisation se confond avec délocalisation et mondialisation est représenté par la traduction collective communautaire (crowdsourcing) qui, à l'image du sous-titrage illégal de séries (beaucoup plus connu sous son appellation anglaise fansubbing) en traduction audiovisuelle, se dresse comme un risque manifeste pour la traduction multimédia professionnelle en mettant à la portée du premier venu la possibilité de traduire à la volée le site qu'il est en train de visiter ou l'application qu'il est en train d'utiliser.

Éblouis par la technologie, les acteurs de la localisation prennent le risque d'oublier qu'ils exercent un métier de communication. Et que la communication reste une affaire humaine.

\section{NOTES}

1. C’est ce que suggère par exemple le site Le Jargon français, hébergé par Linux France. Consultée le 18 août 2010, <http://www.linux-france.org/prj/jargonf/L/localisation.html>.

2. Voir en particulier l'entrée Localisation du Grand dictionnaire terminologique. Consultée le 26 août 2010, <http://www.granddictionnaire.com/btml/fra/r_motclef/index1024_1.asp >.

3. Disponible sur le site de la LISA: $<$ http://www.lisa.org/Localization.61.0.html $>$, consultée le 26 août 2010.

4. The Microsoft Company, Globalization Step by Step. Consultée le 18 août 2010, <http://msdn. microsoft.com/en-us/goglobal/bb688112.aspx>.

5. Online Oxford English Dictionary (2005). Oxford University Press. Consultée le 26 août 2010, $<$ http://dws-sketch.uk.oup.com/cgi-bin/onlineOde/find_lemma.cgi?lemma=locale\&search_type= simple\&include_american=1\&include_deprecated=1\&include_caps=1\&parts_type=all\&caption style=long\&right_column_mode=synonyms $>$.

6. Voir par exemple la page «Internationalization», Python V2.7. The Python Standard Library. Consultée le 18 août 2010, <http://docs.python.org/library/i18n.html>.

7. Voir le modèle présenté, pour la traduction audiovisuelle, par Froeliger, Nicolas et Audinot, Isabelle (à paraître): Marché de la traduction et marché des formations en traduction, ou les conséquences de nos inconséquences, contribution au colloque international Commerce et traduction: pour une approche pluridisciplinaire et plurilingue des jeux et enjeux de la traduction, Université de Nanterre Paris X, 27-29 novembre 2008.

8. Hollan, James (2008): Language and localization industry knows robust growth. TCworld. Novembre-décembre 2008: 11-12. Consultée le 20 août 2010, <www.tekom.de/upload/alg/tcworld_ 608.pdf>.

\section{RÉFÉRENCES}

Beninatto, Renato S. et Kelly, Nataly (2009): Ranking of Top 30 Language Services Companies. Common Sense Advisory, Inc. Consultée le 15 mai 2009, <http://www.commonsenseadvisory.com>. 
Berman, Antoine (1999): La traduction et la lettre, ou l'auberge du lointain. Paris: Seuil.

Coris, Marie (2008): Proximité et délocalisations: le cas du logiciel. Revue d'économie régionale et urbaine. 3:361-380.

DePalma, Donald A. (2004): Business Without Borders: A Strategic Guide to Global Marketing. Chelmsford: Globa Vista Press.

Dohler, Per N. (1997): Facets of Software Localization: A translator's View. The Translation Journal. 1(1). Consultée le 18 août 2010, <http://accurapid.com/journal/softloc.htm>.

Esselink, Bert (1998): A Practical Guide to Software Localization. Amsterdam: John Benjamins.

Esselink, Bert (2000): A Practical Guide to Localization. Amsterdam: John Benjamins.

Esselink, Bert (2003): The Evolution of Localization. The Guide to Localization, supplément de Multilingual Computing and Technology. 14(5):4-7.

Folaron, Debbie et GAmbier, Yves (2007): La localisation: un enjeu de la mondialisation. In: Joanna NowiCKi et Michael Oustinoff, dir. Traduction et mondialisation. Paris: CNRS Éditions, 37-43.

Froeliger, Nicolas (2007): Pourquoi avoir peur de l'informatisation en traduction? Tribune internationale des langues vivantes. 43:40-54.

Gambier, Yves (2006): Mondialisation en cours et traduction. Meta. 51(4):848-853.

Gouadec, Daniel (2002): Profession traducteur. Paris: La Maison du dictionnaire.

GouAdec, Daniel (2003): Le bagage spécifique du localiseur/localisateur - Le vrai «nouveau profil» requis. Meta. 48(4):526-545.

Mehedintu, Anca et Pirvu, Cerasela (dernière mise à jour le 8 décembre 2009): Enterprise Resources Planning Systems: accountancy support. Dematerialization of the documents. MPRA Paper No. 18999. Munich: Munich Personal RePEc Archive. Consultée le 18 août 2010, <http://mpra.ub.uni-muenchen.de/18999/1/MPRA_paper_18999.pdf>.

Meschonnic, Henri (1999): Poétique du traduire. Lagrasse: Verdier.

Meschonnic, Henri (2006): Le sens du langage. In: Marianne Lederer, dir. Le sens en traduction. Paris: Lettres modernes Minard, 127-139.

Nerrière, Jean-Paul (2004-2006): Don't speak English, parlez Globish. Paris: Eyrolles.

Nerrière, Jean-Paul (2005): Découvrez le Globish: L'anglais allégé en 26 étapes. Paris: Éditions d'organisation.

Niosi, Jorge et Tschang, F. Ted (2009): The strategies of Chinese and Indian software multinationals: implications for internationalization theory. Oxford: Oxford University Press.

Pyм, Anthony (2003): Localization and the Changing Role of Linguistics. In: Salah MejRI, Taïeb BAcCouche, André Clas, et al., dir.: Traduire la langue - traduire la culture. Paris: Maisonneuve et Larose; Tunis: Sud Éditions, 295-304.

QUIRION, Jean (2006): La localisation, palimpseste de l'aménagement terminologique? Stratégies d'implantation terminologique et marketing. Meta. 51(4):824-837.

Smith-Ferrier, Guy (2006): NET Internationalization: The Developer's Guide to Building Global Windows and Web Applications. Reading: Addison-Wesley Professional.

St. Amant, Kirk (2005): A Rhetorical Perspective on Localization and International Outsourcing. In: Laura C. Rivero, Jorge Horacio Doorn et Viviana E. Ferraggine, dir. Encyclopedia of Database Technologies and Applications. Hershey: IGI Global Publishing, 570-574.

Vermeer, Hans J. (1996): A Skopos Theory of Translation. Heidelberg: TEXTconTEXT Verlag. 